\title{
The effect of difficulty and execution scores on total ranking during 2019 Rhythmic Gymnastics World Championships
}

\section{Berfin Serdil Örs}

Department of Coaching Education, Faculty of Sport Sciences, Aydin Adnan Menderes University, Aydin, Turkey.

\begin{abstract}
Rhythmic gymnastics (RG) is a competitive sport and competition results include multiple components such as difficulty (D) and execution (E) scores. Both coaches and researchers should consider the components of performance scores separately. The aim of the current study was to identify the most indicative apparatus score on total ranking in the individual all-around finals of 37th RG World Championships (WCh), 2019, Baku, Azerbaijan and to determine the most important $D$ and/or $E$ scores on overall ranking. Total of 312 scores of 24 gymnasts competed at individual all-around finals were analyzed. Correlations among total qualification, total $D$, total $E$, and total apparatus scores of each apparatus were determined by Pearson correlation analysis. Multiple regression analysis was used and model-fitting performance was assessed by coefficient of determination $\left(\mathrm{R}^{2}\right)$. When correlations among total qualification score and total $D$, total $E$ scores of each apparatus were analyzed moderate and strong relationships were found $(p<$ 0.001). The percentages that $D$ and $E$ scores explained the total score ranged between 79.3 and $44 \%$. Total ball $\mathrm{D}$ score explained $79.3 \%$ of the variability in standings and it was the best predictor score. Total ribbon $E$ score was found to be the second effective score on ranking $(71 \%)$. Finally, total hoop $E$ score had the least effect by $44 \%$. Apart from these, two different total score estimation models that trainers/gymnasts may use practically were enhanced during this study.
\end{abstract}

Keywords: Competition scores, score analysis, rhythmic gymnastics, total score estimation model.

E-mail: bsutcu@adu.edu.tr. Tel: +90 25631535 38. Fax: +90 2563153531

\section{INTRODUCTION}

Gymnastics is considered to be one of the oldest sports and International Gymnastics Federation (FIG) was founded in 1881. On the other hand, rhythmic gymnastics (RG) started as a sport event in the 1940s (Gram and Kari, 2020) and it was part of Olympics in 1984 for the first time (Polat and Örs, 2018). This Olympic sport depends on the gymnasts' morphokinetic abilities evaluated on the basis of aesthetic requirements (Zaccagni et al., 2019). RG is a competitive sport and gymnasts perform their routines with music, using different apparatus (rope, hoop, ball, clubs, ribbon) (Hashimoto et al., 2017). Gymnasts must adhere to both quantitative and qualitative criteria specified in the rules called code of points (CoP) (Sierra-Palmeiro et al., 2019). COP is set by FIG RG Technical Committee (Hashimoto et al., 2017) and updated every four years. For the past 20 years, FIG has made numerous changes to the CoP aiming to increase the objectivity of the judges scoring, and to stimulate the development of RG (Sierra-Palmeiro et al., 2019). The last rules were revised after the Rio Olympic Games (2016), and according to the outcomes of competitions in 2017, rules were updated. According to CoP (2017-2020) $D$ judges have two subgroups $\left(D_{1-2}, D_{3-}\right.$ 4) and they evaluate the difficulties [body difficulties (BD), dance steps combinations (S), apparatus difficulties (AD), and dynamic elements with rotations (DER)] and the total $D$ score is sum of the difficulty elements that gymnast perform. On the other hand, execution score $(E)$ also has two subgroups $\left(E_{1-2}, E_{3-4-5-6}\right)$ and can be scored with a maximum of ten points and judges deduct the mistakes of the gymnasts. The final E-score is calculated as sum of the artistic and technical deductions are subtracted from 10.00 points (FIG, 2017). Recent rules indicate that exercises can be scored with no upper limit of difficulty 
score (D) which means gymnasts can perform as many elements as they can perform in their routines. But the 9 highest correctly performed body difficulties will be counted by judges (FIG, 2017).

Rhythmic gymnastics has a complex structure and due to this complexity factors affecting performance are frequently examined in the literature. When the researches on $R G$ literature are examined, it is seen that studies analyzed body difficulties for RG (Cicchella, 2009; Sousa and Lebre, 1996, 1998), in an attempt to understand the competitive model and performance in $R G$, some studies have analyzed the composition of competition routines (Agopyan, 2014; Agopyan and Örs, 2019; Avila-Carvalho et al., 2012). Moreover, anthropometric physiological identifiers of $R G$ performance (Douda et al., 2008) anthropologic factors of performance in RG (Miletić et al., 2004), the effect of physical fitness to the technical E score (Donti et al., 2016) in RG are the mostly studied topics.

In RG, a competition result includes multiple components such as the difficulty level of routines, relationships between movements and music, idea of the routine, and apparatus difficulty (Donti et al., 2016). For this reason, both coaches and researchers should consider the components of performance scores (difficulty and execution) separately, as described in the RG CoP (2017-2020). Determining the most effective score and apparatus on ranking will contribute to coaches and gymnasts, to create the structure of choreographies. It may be an advantage for them to create the structure of choreographies and which apparatus to gain more superiority. Therefore, aim of the current study was to identify the most indicative apparatus score on total ranking of gymnasts in the individual all-around finals of $37^{\text {th }}$ RG World Championships (WCh), 2019 Baku/Azerbaijan and to determine the most important $D$ and/or E scores on overall ranking.

\section{MATERIALS AND METHODS}

\section{Participants}

A hundred and sixteen gymnasts competed at the individual all-around qualification competitions of $37^{\text {th }} R G$ WCh, 2019, Baku/Azerbaijan. After these qualification competitions the first 24 gymnasts competed at individual all-around finals. Research data constituted of D, E and total scores of 24 senior individual gymnasts who competed in the individual all-around finals.

\section{Procedure}

Official performance scores for gymnasts competing in the individual all-around finals of $37^{\text {th }}$ RG WCh, 2019, Baku/Azerbaijan were analyzed. The gymnasts' competition scores were reached from the results book of $37^{\text {th }}$ RG WCh that are published in the FIG's official web page (https://www.gymnastics.sport/site/events/detail.php ?id=13338\#loaded).

Gymnasts competed with all four apparatus during individual all-around finals. The rules governing the determination of the final score are identical for all sessions of competitions. For individual all-around finals the final score of an exercise is established by the addition of the $\mathrm{D}$ score and $\mathrm{E}$ score.

Total of 312 scores were analyzed during the current study. Gymnasts' scores were determined as follows: (1) 24 total hoop D scores (THDS), (2) 24 total hoop E scores (THES), (3) 24 total hoop scores (THS), (4) 24 total ball D scores (TBDS), (5) 24 total ball E scores (TBES), (6) 24 total ball scores (TBS), (7) 24 total clubs D scores (TCDS), (8) 24 total clubs E scores (TCES), (9) 24 total clubs scores (TCS), (10) 24 total ribbon D scores (TRDS), (11) 24 total ribbon E scores (TRES), (12) 24 total ribbon scores (TRS), (13) 24 total qualification scores (TQS: sum of all scores).

\section{Statistical analysis}

The trial version of the SPSS 22.0 (SPSS Inc., Chicago, IL) was used for statistical analysis of the study. Conformity of the data to normal distribution was assessed with the Kolmogorov-Smirnov test. The correlation among total scores and among TQS, total D, total E, total apparatus scores on each apparatus were determined by Pearson correlation analysis. The magnitude of the correlations was classified as trivial for correlation coefficient $(R)<0.1$, low for $R$ between 0.10 and 0.30 , moderate for $R$ between 0.31 and 0.50 , high for $\mathrm{R}$ between 0.51 and 0.70 , very high for $\mathrm{R}$ between 0.71 and 0.90 , nearly perfect for $\mathrm{R}$ between 0.91 and 0.99 , and perfect for $R=1$ (Hopkins, 2000). Multiple regression analysis was applied to build a model between "TQS" and "TBDS, TRES, TCDS, TBES, THES, TRDS". Forward stepwise was used as variable selection method. Modelfitting performance was assessed by coefficient of determination $\left(R^{2}\right)$. Regression coefficients and their standard errors were also given. Mean \pm standard deviation, minimum and maximum were given as descriptive statistics of quantitative variables that fit normal distribution. Significant level was set at $p<$ 0.05 .

\section{RESULTS}

Descriptive statistics of D, E and total scores are given in Table 1.

\section{Total scores}

For the first 24 gymnasts, there were strong correlations 
Table 1. Descriptive statistics of D, E, and total scores for all apparatus.

\begin{tabular}{lccc}
\hline & Mean \pm Standard Deviation & Minimum & Maximum \\
\hline THDS & $12.917 \pm 0.865$ & 11.000 & 14.800 \\
THES & $8.049 \pm 0.526$ & 7.100 & 9.050 \\
THS & $20.947 \pm 1.247$ & 18.150 & 23.800 \\
TBDS & $12.338 \pm 0.943$ & 10.500 & 14.300 \\
TBES & $8.195 \pm 0.616$ & 7.000 & 9.300 \\
TBS & $20.518 \pm 1.429$ & 18.500 & 23.100 \\
TCDS & $13.017 \pm 0.950$ & 10.800 & 14.900 \\
TCES & $7.804 \pm 0.809$ & 5.950 & 9.150 \\
TCS & $20.817 \pm 1.651$ & 16.750 & 24.050 \\
TRDS & $10.725 \pm 1.088$ & 8.400 & 12.800 \\
TRES & $7.968 \pm 0.600$ & 6.400 & 9.150 \\
TRS & $18.678 \pm 1.606$ & 15.450 & 21.650 \\
TQS & $80.955 \pm 5.126$ & 70.850 & 91.400 \\
\hline
\end{tabular}

THDS: Total hoop D score, THES: Total hoop E score, THS: Total hoop score, TBDS: Total ball D score, TBES: Total ball E score, TBS: Total ball score, TCDS: Total clubs D score, TCES: Total clubs E score, TCS: Total clubs score, TRDS: Total ribbon D score, TRES: Total ribbon E score, TRS: Total ribbon score, TQS: Total qualification score.

- magnitude ranging between 0.837 and 0.907 - between TQS and all of the apparatus scores (for all apparatus $p<$ 0.001). TBS had the strongest correlation with TQS (Table 2).

Results of the stepwise multiple regression analysis in predicting the positioning are summarized in Table 3 . For gymnasts, TBS explained $82.2 \%$ of the variability in standings and it was the best predictor score for overall ranking in the finals. The prediction equation for total score was found as follows $(p<0.001)$ :

TQS $_{1}=1.003^{\star}$ TBS $+0.998^{*}$ TRS $+0.998^{*}$ TCS + $1.002^{*}$ THS (Table 3 ).
When the correlations among TQS and total D, total E scores of each apparatus were analyzed moderate and strong relationships were found and TBDS showed the strongest correlation with TQS (Table 4).

Results of the stepwise multiple regression analysis in predicting the positioning are summarized in Table 5. For gymnasts, TBDS explained $79.3 \%$ of the variability in standings and it was the best predictor score for overall ranking in the finals. The prediction equation for TQS was found as follows $(p<0.001)$ :

$\mathrm{TQS}_{2}=1.828^{*} \mathrm{TBDS}+1.792^{*} \mathrm{TRES}+1.310^{*} \mathrm{TCDS}+$ $1.065^{\star}$ TBES + 1.542*THES + 0.699*TRDS (Table 5).

Table 2. The correlations between total ranking score and total apparatus scores.

\begin{tabular}{lcccc}
\hline Variables & THS & TBS & TCS & TRS \\
\hline TQS $r$ & $0.852^{*}$ & $0.907^{*}$ & $0.837^{*}$ & $0.862^{*}$ \\
\hline${ }^{*} p<0.001$. TQS: Total qualification score. & & &
\end{tabular}

Table 3. Multiple regression equation predicting final standing during the $37^{\text {th }}$ RG WCh (total scores).

\begin{tabular}{|c|c|c|c|c|c|c|}
\hline \multirow{2}{*}{ Model } & \multicolumn{2}{|c|}{ Unstandardized coefficients } & \multirow{2}{*}{$\mathbf{R}^{2}$} & \multirow{2}{*}{$t$} & \multirow[b]{2}{*}{$\mathbf{p}$} & \multirow{2}{*}{$\begin{array}{c}\text { Collinearity statistics } \\
\text { VIF }\end{array}$} \\
\hline & B & Std. Error & & & & \\
\hline Constant & -0.026 & 0.080 & & -0.329 & 0.750 & \\
\hline TBS & 1.003 & 0.006 & 82.2 & 159.87 & $<0.001$ & 3.78 \\
\hline TRS & 0.998 & 0.004 & 74.3 & 234.18 & $<0.001$ & 2.21 \\
\hline TCS & 0.998 & 0.004 & 70.1 & 248.60 & $<0.001$ & 2.07 \\
\hline THS & 1.002 & 0.006 & 72.6 & 154.54 & $<0.001$ & 3.08 \\
\hline
\end{tabular}

B: Regression coefficient, Std. Error: Standard error, VIF: Variance inflation factor TBS: Total ball score, TRS: Total ribbon score, TCS: Total clubs score, THS: Total hoop score. 
Table 4. The correlations among total ranking score and total $\mathrm{D}$, total $\mathrm{E}$ scores of each apparatus.

\begin{tabular}{|c|c|c|c|c|c|c|c|c|c|}
\hline & & THDS & THES & TBDS & TBES & TCDS & TCES & TRDS & TRES \\
\hline TQS & $r$ & $0.832^{*}$ & $0.664^{*}$ & $0.890^{*}$ & $0.743^{*}$ & $0.830^{*}$ & $0.724^{\star}$ & $0.784^{*}$ & $0.843^{*}$ \\
\hline
\end{tabular}

${ }^{*} p<0.001$

TQS: Total qualification score.

Table 5. Multiple regression equation predicting final standing during the $37^{\text {th }}$ RG WCh (total $D$, total E scores).

\begin{tabular}{|c|c|c|c|c|c|c|}
\hline \multirow{2}{*}{ Model } & \multicolumn{2}{|c|}{ Unstandardized coefficients } & \multirow{2}{*}{$\mathbf{R}^{2}$} & \multirow{2}{*}{$\mathbf{t}$} & \multirow{2}{*}{$\mathbf{p}$} & \multirow{2}{*}{$\begin{array}{c}\text { Collinearity Statistics } \\
\text { VIF }\end{array}$} \\
\hline & B & Std. Error & & & & \\
\hline Constant & -1.565 & 1.746 & & -0.897 & 0.382 & \\
\hline TBDS & 1.828 & 0.233 & 79.3 & 7.859 & $<0.001$ & 5.297 \\
\hline TRES & 1.792 & 0.430 & 71 & 4.165 & $<0.001$ & 7.344 \\
\hline TCDS & 1.310 & 0.181 & 68.8 & 7.229 & $<0.001$ & 3.269 \\
\hline TBES & 1.065 & 0.241 & 55.2 & 4.429 & $<0.001$ & 2.418 \\
\hline THES & 1.542 & 0.324 & 44 & 4.765 & $<0.001$ & 3.194 \\
\hline TRDS & 0.699 & 0.187 & 61.5 & 3.732 & 0.002 & 4.571 \\
\hline
\end{tabular}

B: Regression coefficient, Std. Error: Standard error, VIF: Variance inflation factor

TBDS: Total ball D score, TRES: Total ribbon E score, TCDS: Total clubs D score, TBES: Total ball E score, THES: Total hoop E score, TRDS: Total ribbon D score.

\section{DISCUSSION}

The aim of the current study was to determine the most effective apparatus score on ranking of gymnasts in the individual all-around finals of $37^{\text {th }}$ RG WCh, 2019, Baku/Azerbaijan. In accordance with this aim, the main finding of this study was that TBS showed the strongest correlation with TQS (82\%). In accordance with these findings, the most important apparatus score predicting the ranking of first 24 gymnasts was ball.

Moreover, TBDS was the most correlated score to TQS among other D and E scores. TQS was explained $79.3 \%$ by TBDS. On the other hand, the predictor that was less influenced by the technique of the gymnasts was the score gained on TCS. And, THES explained $66.4 \%$ of TQS by the least percentage among other $D$ and $E$ scores. Rhythmic gymnasts perform with 5 apparatuses (rope, hoop, ball, clubs, and ribbon). These five apparatus can be divided into 2 groups: (1) rigid (hoop, ball, and clubs), (2) soft (ribbon and rope) (Jastrjembskaia and Titov, 1999). On individual program, the competition includes the use of four of the five apparatus used in this sport event and, in turn, one of them is left out: for example, during this four-year Olympic period, the rope was kept out (Senatore et al., 2019). Gymnasts need more endurance for their clubs routines than other apparatus. On the other hand, ribbon, as it is six meters long, is considered to be technically difficult to perform compared to other apparatus (Polat and Örs, 2018). In parallel with difficult execution of ribbon, gymnasts' probability of making mistakes gets higher and this results that gymnasts have lower $E$ scores with ribbon. Considering the final scores in the present study, TBS and THS seem to have the biggest effect on predicting the TQS. Moreover, TRS and TCS have the same effect on TQS by 0.998 coefficients. Also, hoop and ball have better $E$ scores. This may be the reason that TBS and THS have higher effects than TCS and TRS. Consequently, these results seem to support the literature.

Due to the changes in CoP, skill complexity of gymnasts has been increasing and routines on each apparatus have been developing (Irwin et al., 2005). As a result of this increase and development, to understand the technique that gymnasts need and optimize the training according to these requirements, it is essential to understand the diversity of scores during top level competitions. In the literature it is reported that penalties often occur in competitions early in the season, and they decrease as perfection is achieved (Hashimoto et al., 2017). In the current study, THS ranged from 18.150 to 23.800 points, TBS ranged from 18.500 to 23.100 , TCS ranged from 16.750 to 24.050 and TRS ranged from 15.450 to 21.650 . According to these scores it is clear that gymnasts had the lowest score with ribbon and the best score with clubs. While hoop, ball, and ribbon are sole apparatus, clubs are paired apparatus. Gymnasts have two clubs to perform their routines and it can be said that clubs are a bit difficult to perform with. On the other hand, TCDS was found to be the highest score (14.900) among other $D$ and $E$ scores. This can be the reason that gymnasts can perform more variety of 
apparatus elements (for example: dynamic elements with rotation) with clubs. As a result, possibility of making mistakes increase and $E$ scores get lower. This may be the reason why TCES was lower during this study. Barely, as $D$ and $E$ scores were not analyzed in subgroups $\left(D_{1-2}, D_{3-4}, E_{1-2}, E_{3-4-5-6}\right)$ during this study, future analysis can also evaluate the correlations among total score with subgroups of $D$ and $E$ score.

In international competitions top level gymnasts' apparatus scores are around get 18.000 points (for one apparatus) and Yana Kudryavtseva, at the WCh (2013, Kiev) were scored 18.700 for her clubs routine and 73.866 for sum of all four apparatus and this was a World record (Hashimoto et al., 2017). E scores of top gymnasts in the World Championship are over 9.000 points for all four apparatus (Hashimoto et al., 2017) and in the current study gymnasts had minimum of 5.950 points for TCES and maximum of 9.300 points for TBES. These results seem to be different than reported in the literature. Moreover, Leandro et al. (2016) reported top twenty-four rhythmic gymnasts competing at the RG WCh (2013) had an average of 8.42 points for D score. In the present study, rhythmic gymnasts had an average of 12.25 point for D scores. FIG RG technical committee updated the rules after the competitions in 2017 and according to the latest rules $D$ score do not have an upper limit any more (before gymnasts could perform $D$ elements up to 10.000) and gymnasts can perform as many elements as they can. With these changes in the rules recently, gymnasts started to get $D$ scores over 10.000 points. So, this increase in D scores as an outcome gymnast started to achieve higher scores in total scores too (more than 20.000). These changes may be the reason for the differences found in the results of the currents study and the literature.

As gymnasts perform a lot of body and apparatus difficulty elements in their routines, this rapid increase in difficulty may have contributed to the results of the current study. Based on the RG judging system, gymnasts are judged separately for $D$ and $E$ scores and this gives gymnasts direct opportunity to raise their scores by increasing the difficulty of the elements in their routines (Irwin et al., 2007). RG routines have been improving rapidly and gymnasts have started to perform a greater number of body and apparatus difficulty elements. With recent changes in the CoP, gymnasts can perform as many difficulty elements as they wish and by performing more elements, they have started to reach very high scores such as 23.00 and more. As execution scores have an upper limit, to rank in the top, gymnasts have started to perform higher $D$ scores and as seen in the results of the current study TBDS has the biggest effect on predicting the TQS by 1.828 coefficient.

\section{RECOMMENDATIONS}

Rhythmic gymnastics has a rapid progress and with this rapid progress, judging the routines has become more challenging. Gymnasts have started to perform many difficulty elements with this progress nowadays; RG has some judging/scoring issues that put this beautiful sport into risk. Recently, it has been announced that there is a risk of being excluded from the Olympics. FIG has started to organize congress and analyze the RG competitions to solve out these problems. RG is as an aesthetic event, even though it has rules, the routines include dancing elements, artistry and art. But, with recent technical developments of gymnasts and changes in the rules, routines have started to include more difficulty elements than the artistic component of the sport. As seen from the results of the current study, each score does not have the same effect on ranking and difficulty scores seem to have a bigger effect. For this reason, coaches should focus on the difficulty level of gymnasts. Besides, as a strategy, coaches may increase their trainings further, especially with ribbon which had the lowest impact. But, to protect the beauty of RG, some changes in the rules should take place.

Only senior individual gymnasts were considered in the current study, similar studies including $D$ and $E$ scores of individual juniors and/or groups will contribute to both coaches and literature. Moreover, determining the $D$ and $E$ scores in subgroups and investigating the relationships with ranking will benefit the literature.

\section{CONCLUSION}

The technical ability of gymnasts both with body and apparatus has an influence on qualification score and rankings. In this study, TBS and TBDS were found to have the biggest effect on TQS. As it is seen from the results; each apparatus and each score component do not have the same effect on qualification. As our results point out that TBDS has one of the biggest effects on rankings. For these reasons; research consisting $R G$ and analyzing RG scores should continue and some effective changes in the rules should take place to protect the beauty and elegancy of the sport and gymnasts with different technical levels should be evaluated not only with difficulty scores but also with their artistic and execution abilities.

\section{REFERENCES}

Agopyan, A. (2014). Analysis of body movement difficulties of individual elite rhythmic gymnasts at London 2012 Olympic games finals. Middle - East Journal of Scientific Research, 19(12): 15541565.

Agopyan, A., and Örs, B. S. (2019). An analysis of variations in body movement difficulty of 2016 Olympic Games rhythmic gymnast candidates. International Journal of Performance Analysis in Sport, 19(3): 417-434.

Avila-Carvalho, L., Palomera, M. da L., Klentrou, P., and Lebre, E. (2012). Analysis of the technical content of elite rhythmic gymnastics group routines. The Open Sports Sciences Journal, 5(1), 146-153.

Cicchella, A. (2009). Kinematics analysis of selected rhytmic gymnastic 
leaps. Journal of Human Sport and Exercise, 4(1): 40-47.

Donti, O., Bogdanis, G. C., Kritikou, M., Donti, A., and Theodorakou, K. (2016). The relative contribution of physical fitness to the technical execution score in youth rhythmic gymnastics. Journal of Human Kinetics, 50(2): 143-152.

Douda, H. T., Toubekis, A. G., Avloniti, A. A., and Tokmakidis, S. P. (2008). Physiological and anthropometric determinants of rhythmic gymnastics performance. International Journal of Sports Physiology and Performance, 3(1): 41-54.

FIG (2017). Code de Pointage Gymnastique Rythmique: 2017- 2020 (F. I. De Gymnastique-FIG (Ed.); FIG, comit).

Gram, M. C. D., and Kari, B. (2020). High level rhythmic gymnasts and urinary incontinence: Prevalence, risk factors, and influence on performance. Scandinavian Journal of Medicine and Science in Sports, 30(1): 159-165.

Hashimoto, M., Kida, N., and Nomura, T. (2017). Characteristics of women's rhythmic gymnastics from the perspective of "body difficulty" and performance time. Advances in Physical Education, 7(3): 260273.

Hopkins, W. G. (2000). Measures of reliability in sports medicine and science. Sports Medicine, 30(1): 1-15.

Irwin, G., Hanton, S., and Kerwin, D. G. (2005). The conceptual process of skill progression development in artistic gymnastics. Journal of Sports Sciences, 23(10): 1089-1099.

Irwin, G., Hanton, S., and Kerwin, D. G. (2007). The conceptual process of skill progression development in artistic gymnastics. Journal of Sport Sciences, 23(10): 1089-1099.

Jastrjembskaia, N., and Titov, Y. (1999). Rhythmic Gymnastics. Human Kinetics.

Leandro, C., Ávila-Carvalho, L., Sierra-Palmeiro, E., and Bobo-Arce, M. (2016). Departure difficulty score vs final difficulty score. The effect of performance in elite rhythmic gymnastics. Athens Journal of Sports, 3(3): 169-177.

Miletić, D., Katić, R., and Maleš, B. (2004). Some anthropologic factors of performance in rhythmic gymnastics novices. Collegium Antropologicum, 28(2): 727-737.

Polat, S. C.., and Örs, B. S. (2018). Rhythmic Gymnastics (1st Ed.). Gazi Publishing Company.

Senatore, B., Valentini, M., Federici, A., and D'elia, F. (2019). Pilot case study on rhythmic gymnastics and dyslexia. Journal of Human Sport and Exercise, 14(Proc2): S198-S205.
Sierra-Palmeiro, E., Bobo-Arce, M., Pérez-Ferreirós, A., and Fernández-Villarino, M. A. (2019). Longitudinal study of individual exercises in elite rhythmic gymnastics. Frontiers in Psychology, 10: 1496.

Sousa, F., and Lebre, E. (1996). Biomechanical analysis of two different jumps in rhythmic sports gymnastics (RSG). XIV International Symposium on Biomechanics in Sports, 416-419.

Sousa, F., and Lebre, E. (1998). Biomechanics of Jumps in Rhythmic Sport Gymnastics (RSG) a Kinematic Analysis of the Principal Jumps in RSG. 255-258.

Zaccagni, L., Rinaldo, N., and Gualdi-Russo, E. (2019). Anthropometric indicators of body image dissatisfaction and perception inconsistency in young rhythmic gymnastics. Asian Journal of Sports Medicine, 10(4): e87871.

Citation: Örs, B. S. (2020). The effect of difficulty and execution scores on total ranking during 2019 Rhythmic Gymnastics World Championships. African Educational Research Journal, 8(1): S37S42. 